\title{
ON THE H. LEWY EXTENSION PHENOMENON
}

\author{
BY \\ RICARDO NIRENBERG
}

\begin{abstract}
We prove local approximation and extension theorems for $C^{\infty}$ submanifolds $M$ of $C^{n}$ (CR submanifolds). Under some conditions on $M$, any smooth solution of the induced Cauchy-Riemann equations can be extended holomorphically to bigger (and sometimes open) sets.
\end{abstract}

1. Introduction. A characteristic feature of the theory of functions of several complex variables is the holomorphic extendibility of functions from a set to a bigger set. This phenomenon is intimately related to the vanishing of certain spaces of cohomology with compact support. The first example of this is the well-known theorem of Hartogs: any function defined and holomorphic on a neighborhood of the boundary of a ball in $\boldsymbol{C}^{n}(n>1)$ can be extended holomorphically to the interior of the ball. A stronger form of this result was given by Bochner (see [7, Theorem 2.3.2']): if $D$ is a bounded open set in $C^{n}(n>1)$ such that $C^{n}-\bar{D}$ is connected and $D$ has a $C^{4}$ boundary bd $D$, then any $C^{4}$ function on bd $D$ satisfying the partial differential equations on bd $D$ indexed by the Cauchy-Riemann equations in $C^{n}$ can be extended holomorphically to $D$. In [9], H. Lewy proved a local version of this theorem (see also [7, Theorem 2.6.13]): if the Levi form of a smooth real hypersurface does not vanish at a point $z_{0}$, then smooth solutions of the induced Cauchy-Riemann equations on a sufficiently small neighborhood of $z_{0}$ can be holomorphically extended to at least one side of the hypersurface. Thus we see that the extendibility property for a hypersurface does not depend on its bounding an open domain, but is already present on any small portion of it. Later in [10] H. Lewy gave an example of a piece of a 4-real dimensional submanifold $M$ in $C^{3}$ with the property that any smooth solution of the induced Cauchy-Riemann equations on it can be extended to a holomorphic function on an open set of $\boldsymbol{C}^{3}$ whose boundary contains $M$.

From then on, much work has been done on the local extendibility properties of real submanifolds $M$ of $C^{n}$ with real dimension less than $2 n-1$, but always in the setup of Hartogs' result, that is, one looks at functions which are holomorphic on some open neighborhood of $M$ in $C^{n}$, and tries to extend them to a fixed open set of $C^{n}$. In other words, one extends germs of holomorphic functions on $M$. The pioneer work in this direction was done by Bishop [3]. Further results are found in

Received by the editors August 3, 1971.

AMS 1969 subject classifications. Primary 3225, 3270.

Research supported by NSF grant GP-24182 at the University of Wisconsin, Madison, Wisconsin.

Copyright (C) 1972, American Mathematical Society 
[4], [5], [16], [17] and [18]. A brief account of this area of research can be found in [19].

In this paper we adopt the point of view of Bochner's and Lewy's results, trying to extend smooth solutions of the induced Cauchy-Riemann equations on a piece of real smooth submanifold of $\boldsymbol{C}^{n}$. The procedure is to prove first an approximation theorem: such solutions can be uniformly approximated on $M$ (locally) by germs of holomorphic functions on $M$, and then use the results already known for the extension of germs. Thus the theory of extension from Lewy's point of view is reduced to the theory for germs.

We consider $M$ to be a CR submanifold (see $\$ 2$ ) and the approximation theorem is proved under certain convexity conditions on $M$. Briefly, we will assume that either there is a real hypersurface passing through $M$ which is strongly pseudoconvex at $z_{0} \in M$, or that there is a real hypersurface through $M$ whose Levi form restricted to $H_{0}(M)$ (the space of complex tangent vectors to $M$ at $z_{0}$ ) has at least one positive and at least one negative eigenvalue. In the simplest nontrivial case of $M^{4}$ in $C^{3}$, this means that there is a real hypersurface through $M^{4}$ whose Levi form restricted to $H_{0}$ is not zero. Under these conditions we can extend to the same sets as in the extension of germs. (For hypersurfaces, no convexity conditions are needed in the approximation theorem (see [13]).)

In $\$ 2$ we present an account of the basic properties of CR submanifolds of $\boldsymbol{C}^{n}$ and we give some examples. In $\$ 3$ we put together the results on $\bar{\partial}$-cohomology with compact support that are needed, and we prove that if a real hypersurface in $\boldsymbol{C}^{n}$ satisfies certain convexity conditions, the equation $\bar{\partial} f=g$ can be solved locally in $L^{2}$ with 0 boundary values on a piece of the hypersurface. When $g$ is a $(0,1)$ form this is due to Lewy [9]; see also Hörmander [7, Theorem 2.6.13]. The idea of the proof for forms of other types is due to A. Andreotti. The main results of the paper are proved in $\S 4$. For an account of the notions of holomorphic convexity of sets and of some of the problems in this connection, we refer the reader to [14], [15] and [19].

A weaker result for the case $\mathrm{CR}$ codim $M=2$ was published without the complete proofs in [12].

\section{CR submanifolds of $\boldsymbol{C}^{n}$.}

2.1. Definitions. Let $M$ be a real $C^{\infty}$ submanifold of $C^{n}$, of real dimension $2 n-k$. Let $T(M)$ be the tangent bundle to $M, T_{x}(M)$ its fiber at $x \in M$, and consider the complexified bundle

$$
T^{c}(M)=T(M) \otimes_{R} C .
$$

$T^{c}(M)$ is a subbundle of $T^{c}\left(C^{n}\right)$. On the latter we consider the operator $J$ given by the complex structure on $C^{n}$. Set

$$
H_{x}(M)=\left\{\xi \in T_{x}^{c}(M) ; J(\xi) \in T_{x}^{c}(M)\right\} ; \quad x \in M .
$$


We write $2 m(x)=\operatorname{dim}_{C} H_{x}(M)$. If $m(x)$ is a constant on connected components of $M$, we say that $M$ is a CR submanifold of $C^{n}$, and for a connected CR submanifold $M$ we write $m=\mathrm{CR} \operatorname{dim} M$. For a CR submanifold $M, H_{x}(M)$ are the fibers of a vector bundle $H(M)$ on $M$, whose sections are called complex vector fields on $M$. We will consider only connected CR submanifolds of $\boldsymbol{C}^{n}$, and will assume in general that $m>0$. (If $m=0, M$ is said to be totally real.) Let

$$
\begin{aligned}
& l^{\prime}=\operatorname{dim}_{R} M-2 \mathrm{CR} \operatorname{dim} M=2(n-m)-k=2 \mathrm{CR} \operatorname{codim} M-\operatorname{codim}_{R} M, \\
& l^{\prime}=2 l-k .
\end{aligned}
$$

Then $l^{\prime}$ is the fiber dimension of $T^{c}(M) / H(M)$. We will assume that $l^{\prime}>0$, for otherwise $M$ is a complex submanifold of $C^{n}$. It is always true that $l^{\prime} \leqq k$. If $k \leqq n$ and $l^{\prime}=k$, we say that $M$ is a generic CR submanifold of $C^{n}$. In the case $k>n$, we say that $M$ is generic if $l^{\prime}=2 n-k$, that is, if $m=0$. In any case, a complex submanifold is generic if and only if it is a point or $\boldsymbol{C}^{n}$ itself.

A vector field $\zeta$ on $M$ is said to be of type $(1,0)$ if $J(\zeta)=(-1)^{1 / 2} \zeta$, and of type $(0,1)$ if $J(\zeta)=-(-1)^{1 / 2} \zeta$. Given a point $x \in M$ it is easy to see that there is a neighborhood $U$ of $x$ in $M$ and a basis for sections of $H(M)$ on $U$ of the form

$$
\left\{\zeta_{1}, \ldots, \zeta_{m}, \zeta_{1}, \ldots, \zeta_{m}\right\}
$$

where $\zeta_{i}, i=1, \ldots, m$, are complex vector fields on $U$ of type $(1,0)$, and therefore $\xi_{i}$ are of type $(0,1)$.

Let $\mathscr{L} H(M)$ stand for the complex Lie algebra of vector fields generated by the sections of $H(M)$. If $\operatorname{dim}_{C} \mathscr{L} H(M)$ is a constant, we set $e(M)=\operatorname{dim}_{C} \mathscr{L} H(M)$ $-\operatorname{dim}_{C} H(M)$.

2.2. Defining systems. Let $\rho_{1}, \ldots, \rho_{k}$ be real valued $C^{\infty}$ functions defined on some open neighborhood $D$ of $M$ in $C^{n}$ such that

(i) $M=\left\{x \in D ; \rho_{1}(x)=\cdots=\rho_{k}(x)=0\right\}$,

(ii) $d \rho_{1} \wedge \cdots \wedge d \rho_{k} \neq 0$ on $M$.

Such a system of functions is called a defining system for $M$ (locally it always exists). If $\left\{\rho_{1}, \ldots, \rho_{k}\right\}$ is a defining system for $M$ near $x \in M$, the most general defining system for $M$ near $x$ is obtained by taking

$$
\rho_{i}^{\prime}=\sum_{j=1}^{k} f_{i j} \rho_{j}, \quad i=1, \ldots, k,
$$

where $f_{i j}$ are defined and $C^{\infty}$ on some neighborhood of $x$ in $C^{n}$, and $\operatorname{det}\left(f_{i j}\right) \neq 0$ on $M$.

Let $\left\{\rho_{1}, \ldots, \rho_{k}\right\}$ be any defining system for $M$ near $x \in M$. Then for a vector $\xi \in C^{2 n}$ we have

$$
\xi \in T_{x}^{c}(M) \Leftrightarrow d \rho_{i}^{(x)}(\xi)=0, \quad i=1, \ldots, k .
$$


LEMMA 2.2.1. Let $l$ be as in (2.1.2). If $\left\{\rho_{1}, \ldots, \rho_{k}\right\}$ is a defining system for $M$ near $x \in M$, we can find l of the $\rho$ 's (which after reordering we assume are $\rho_{1}, \ldots, \rho_{l}$ ) such that they form a maximal subset for which $\partial \rho_{1} \wedge \cdots \wedge \partial \rho_{l} \neq 0$ on $M$, where we have put $2 \partial=d-(-1)^{1 / 2} J d$ and $2 \bar{\partial}=d+(-1)^{1 / 2} J d$. Furthermore, for $\xi \in C^{2 n}, x \in M$ we have

$$
\xi \in H_{x}(M) \Leftrightarrow \partial \rho_{i}(x)(\xi)=\bar{\partial} \rho_{i}(x)(\xi)=0, \quad i=1, \ldots, l .
$$

The proof is straightforward and is left to the reader.

2.3. The induced $\bar{\partial}$-operator. Let $M$ be a CR submanifold of $C^{n}$ and let $E^{(1,0)}=E$ be the bundle whose sections are the complex vector fields of type $(1,0)$, and similarly for $E^{(0,1)}=\bar{E}$. Let $E^{*}$ be the bundle dual to $E$, and $\bar{E}^{*}$ the dual of $\bar{E}$. We consider the bundles of differential forms on $M: D^{(p, q)}=E^{* p} \wedge \bar{E}^{* q}$, where $E^{p}$ stands for the $p$ th exterior power of $E$. Then $D^{(p, q)}$ is a subbundle of the bundle of $p+q$ forms on $M$. We also write $D^{(p, q)}$ for the space of sections of this bundle.

Consider the operator $\bar{\partial}_{M}: D^{(p, q)} \rightarrow D^{(p, q+1)}$ defined by taking $d \varphi$ for $\varphi \in D^{(p, q)}$ and then projecting it on $D^{(p, q+1)}$. Then we have

$$
\bar{\partial}_{M}^{2}=0 \text {. }
$$

We can therefore define the cohomology spaces

$$
H^{(p, q)}=\left\{\varphi \in D^{(p, q)} ; \bar{\partial}_{M} \varphi=0\right\} / \bar{\partial}_{M}\left(D^{(p, q-1)}\right) .
$$

Another way of viewing this locally is the following: take a defining system for $M,\left\{\rho_{1}, \ldots, \rho_{k}\right\}$, and let $l$ be as in (2.1.2). Let $A^{(p, q)}$ be the sheaf of germs on $M$ of $C^{\infty}$ forms of type $(p, q)$ in $C^{n}$. Consider the form, in $A^{(0, l)}$,

$$
w=\bar{\partial} \rho_{1} \wedge \cdots \wedge \bar{\partial} \rho_{l} .
$$

Then we define the ideals (of forms "normal" at $M$ (see [8])):

$$
N^{(p, q)}=\left\{\varphi \in A^{(p, q)} ; \varphi \wedge w=0 \text { on } M\right\} \subset A^{(p, q)} .
$$

Then we have

$$
D^{(p, q)}=\Gamma\left(A^{(p, q)}, M\right) / \Gamma\left(N^{(p, q)}, M\right) .
$$

Also, the operator $\bar{\partial}$ in $C^{n}$ induces maps

$$
\bar{\partial}: A^{(p, q)} \rightarrow A^{(p, q+1)}, \quad \bar{\partial}: N^{(p, q)} \rightarrow N^{(p, q+1)},
$$

because $\varphi \in N^{(p, q)}$ implies that

$$
\varphi=\sum_{i=1}^{l} \psi_{i} \bar{\partial} \rho_{i}+\sum_{i=1}^{k} \rho_{i} \varphi_{i}
$$

with $\varphi_{i} \in A^{(p, q)}, \psi_{i} \in A^{(p, q-1)}$. Hence $\bar{\partial} \varphi \wedge w=0$ on $M$. As a consequence, $\bar{\partial}$ induces a mapping of the quotients $\bar{\partial}_{M}: D^{(p, q)} \rightarrow D^{(p, q+1)}$, which is the same as the mapping previously defined. 
In particular, a complex-valued $C^{\infty}$ function $f$ on $M$ for which $\bar{\partial}_{M}(f)=0$ will be called a CR function on $M$. It is obvious that if $f \in C^{\infty}(M)$ can be continued holomorphically and $C^{\infty}$ to an open set $V$ of some complex variety such that $M$ lies in the boundary of $V$, then $f$ is a CR function on $M$.

We will need the following algebraic result:

Proposition 2.3.1. If $\varphi \in N^{(p, q)}$, if $r>0$ is a natural number, and $\bar{\partial} \varphi$ vanishes on $M$ to order $r-1$, there is an element $\psi \in A^{(p, q-1)}$ with $\psi \mid M=0$ such that $\varphi+\bar{\partial} \psi$ vanishes on $M$ to order $r$.

For the proof we refer to [13], where the case $\varphi=\bar{\partial} f, f \in A^{(0,0)}$ is considered. However, the argument for the general case goes just along the same lines.

REMARK 2.3.2. By using the following result: let $K$ be closed in $R^{n}$, and let $f \in C^{m}\left(R^{n}\right)$ be such that $D^{k} f(x)=0$ for $x \in K,|k| \leqq m$; then there is a sequence $\left\{f_{v}\right\} \subset C^{\infty}\left(R^{n}\right)$ such that $f_{v} \equiv 0$ on some neighborhood $V_{v}$ of $K$ for each $\nu$, and $f_{v}$ converges to $f$ in $C^{m}\left(R^{n}\right)$ with the topology of uniform convergence of the derivatives up to order $m$ on compact subsets (see [11]), one can prove easily that we can replace in the above proposition the orders $r$ and $r-1$ by order $\infty$. (This is the "soft" part of Whitney's extension theorem.)

Corollary 2.3.3. Let $f \in C^{\infty}(M)$ be such that $\bar{\partial}_{M} f=0$. Then we can find a $C^{\infty}$ extension $\hat{f}$ of $f$ to $C^{n}$ such that $\bar{\partial} \hat{f}$ vanishes on $M$ to a given order $r$ (or even to order $\infty$ by the above remark).

2.4. $C R$ regular defining systems. Let $M$ be a CR submanifold of $C^{n}$ and let $\left\{\rho_{1}, \ldots, \rho_{k}\right\}$ be a defining system for $M$. Let $V$ be an open subset of $M$ and $U$ an open subset of $C^{n}$ with $U \cap M=V$. For $t=\left(t_{1}, \ldots, t_{k}\right) \in R^{k}$ close enough to the origin, we define the $C^{\infty}$ submanifolds: $V(t)=\left\{x \in U ; \rho_{i}(x)=t_{i}, i=1, \ldots, k\right\}$, so that $V(0)=V$.

Definition 2.4.1. We say that the defining system $\left\{\rho_{1}, \ldots, \rho_{k}\right\}$ is CR regular on $V \subset M$ if there is an open set $U \subset C^{n}$ with $U \cap M=V$, and an open neighborhood $W$ of the origin in $R^{k}$, and a $C^{\infty}$ diffeomorphism $\Phi: W \times V \rightarrow U$ such that

(i) $\Phi(0)=\Phi \mid\{0\} \times V=$ Identity on $V$.

(ii) $\Phi(t)=\Phi \mid\{t\} \times V$ is an isomorphism of the CR structures

of the CR submanifolds $V$ and $V(t)$ for every $t \in W$. (We assume

that $V(t)$ is a CR submanifold for $t \in W$.)

The notion of $\mathrm{CR}$ isomorphism is the natural one.

Observe that $\mathrm{CR}$ regularity is invariant under holomorphic changes of coordinates. Also for any CR submanifold $M$ and every point $x \in M$ there are CR regular defining systems for $M$ on any neighborhood of $x$ in $M$ which is small enough. For there is a neighborhood $U$ of $x$ in $C^{n}$ such that if $\left\{x_{1}, \ldots, x_{2 n}\right\}$ are the real coordinates in $C^{n}$, we can write

$$
M \cap U=\left\{x \in U ; x_{i}-g_{i}\left(x_{k+1}, \ldots, x_{2 n}\right)=0 ; i=1, \ldots, k\right\},
$$


where $g_{i} \in C^{\infty}(U)$. Since translations are holomorphic diffeomorphisms, and a fortiori CR isomorphisms, the system $\rho_{i}=x_{i}-g_{i}$ is indeed CR regular on $U \cap M$.

The reason for considering $C R$ regular systems is that with respect to them a $\mathrm{CR}$ function $f$ satisfies the following strong condition:

Lemma 2.4.2. Let $\left\{\rho_{1}, \ldots, \rho_{k}\right\}$ be a $C R$ regular defining system for $M$ on $V \subset M$, and let $l$ be as in (2.1.2). Then there is an open set $U$ in $C^{n}$ with $U \cap M=V$ such that every $C R$ function $f$ on $V$ can be extended to a $C^{\infty}$ function $\hat{f}$ on $U$ satisfying $\bar{\partial} \hat{f} \wedge w=0$ on $U$, where $w$ is given by (2.3.2).

Proof. We can take $W$ in Definition 2.4.1 so that on $\Phi(W \times V)$ we still have $w \neq 0$. Then we define $\hat{f}$ on $U=\Phi(W \times V)$ as follows:

$$
\hat{f} \mid V(t)=f \circ \Phi^{-1}(t) \text { for } t \in W .
$$

Then $\hat{f}$ is a CR function on each $V(t), t \in W$, and therefore $\bar{\partial} \hat{f} \wedge w=0$ identically on $U$. Q.E.D.

By putting Corollary 2.3.3 and Lemma 2.4.2 together it is easy to get the following

Corollary 2.4.3. Let $\left\{\rho_{1}, \ldots, \rho_{k}\right\}$ be a $C R$ regular defining system for $M$ on $V$. Then there is an open set $U$ in $C^{n}$ with $U \cap M=V$ such that for any $C R$ function $f$ and any $r=$ natural number or $\infty$, we can find a $C^{\infty}$ function $\hat{f}$ on $U$ satisfying

(i) $\hat{f}|V=f| V$,

(ii) $\bar{\partial} \hat{f} \wedge w=0$ on $U$,

(iii) $\bar{\partial} \hat{f}$ vanishes on $\hat{M}$ to order $r$, where $\hat{M}=\left\{\rho_{1}=\cdots=\rho_{l}=0\right\} \supset M$.

We will also need the following

LEMMA 2.4.4. If $M$ is a $C R$ submanifold of $C R$ dimension $m$ in $C^{n}$, and there is a real $C^{\infty}$ hypersurface $\{\rho=0\}$ containing $M$ such that the Levi form of $\rho$ restricted to $H_{x}(M)$ has $s$ positive eigenvalues at $x \in M$, then there is a defining system $\left\{\rho_{1}, \ldots, \rho_{k}\right\}$ for $M$ which is $C R$ regular on some neighborhood of $x$ and such that the Levi form of every $\rho_{i}$ has $n-m+s=l+s$ positive eigenvalues on some neighborhood of $x$ in $C^{n}$.

Proof. After a holomorphic change of coordinates, we can assume that $x$ is the origin, $x_{1}, y_{1}, \ldots, x_{m}, y_{m}, x_{m+1}, \ldots, x_{m+l}$ are tangential to $M$ at 0 and $y_{m+1}, \ldots$, $y_{m+l}, x_{m+l+1}, y_{m+l+1}, \ldots, x_{n}, y_{n}$ are normal to $M$ at 0 , where $z_{i}=x_{i}+(-1)^{1 / 2} y_{i}$. Furthermore, we have on some neighborhood $V$ of 0 in $M$

$$
\begin{aligned}
& y_{i}=\varphi_{i}\left(x_{1}, y_{1}, \ldots, x_{m}, y_{m}, x_{m+1}, \ldots, x_{m+l}\right), \quad i=m+1, \ldots, n ; \\
& x_{i}=\psi_{i}\left(x_{1}, y_{1}, \ldots, x_{m}, y_{m}, x_{m+1}, \ldots, x_{m+l}\right), \quad i=m+l+1, \ldots, n ;
\end{aligned}
$$

where $\varphi_{i}$ and $\psi_{i}$ vanish at 0 to order two and are $C^{\infty}$ on some neighborhood of 0 . The hypersurface of the statement can be written as, say,

$$
y_{n}=\varphi\left(x_{1}, y_{1}, \ldots, x_{n-1}, y_{n-1}, x_{n}\right)
$$


where $\varphi$ vanishes at 0 to order 2. Then replacing in (2.4.5) the variables $x_{i}$ $(i=m+l+1, \ldots, n)$ and $y_{i}(i=m+1, \ldots, n-1)$ by the expressions (2.4.4), we still get a function

$$
y_{n}=\hat{\varphi}\left(x_{1}, y_{1}, \ldots, x_{m}, y_{m}, x_{m+1}, \ldots, x_{m+l}\right)
$$

whose Levi form restricted to $H_{x}(M)$ has $s$ positive eigenvalues. Now it is easy to see that the system

$$
\begin{array}{ll}
y_{i}-\varphi_{i}+A\left(y_{n}-\hat{\varphi}\right), & i=m+1, \ldots, n-1 ; \\
x_{i}-\psi_{i}+A\left(y_{n}-\hat{\varphi}\right), & i=m+l+1, \ldots, n ; \\
y_{n}-\hat{\varphi} ; &
\end{array}
$$

is a CR regular defining system on some neighborhood of $x$ in $M$, and that if the real number $A>0$ is big enough, all its functions have a Levi form with $s$ positive eigenvalues when restricted to $H_{x}(M)$. Calling $\rho_{j}, j=1, \ldots, k$, the functions in (2.4.6), we now form

$$
\rho_{i}^{\prime}=\sum_{j=1}^{k} a_{i j} \cdot \exp \left(A_{j} \rho_{j}\right) \cdot \rho_{j},
$$

where $A_{j}$ are real numbers and $\left(a_{i j}\right)$ is a nonsingular real matrix with all $a_{i j}>0$. Then an easy calculation shows that $\left\{\rho_{1}^{\prime}, \ldots, \rho_{k}^{\prime}\right\}$ is a CR regular defining system on some neighborhood of $x$ in $M$, and that if the $A_{j}$ are chosen big enough, the Levi form of each of the functions $\rho_{i}^{\prime}$ will have $s+n-m$ positive eigenvalues on $C^{n}$. Q.E.D.

2.5. Examples. As stated in $\$ 2.1$, we are interested in CR submanifolds $M$ of $C^{n}$ for which $l^{\prime}>0$ ( $M$ is not a complex submanifold), and $0<m<n-1$ ( $M$ is not totally real and it is not a real hypersurface: the approximation theorem of $\$ 4$ in those two cases was proved in [13]). The simplest case is when $M$ is Levi flat, that is, when $e(M)$ (see $\$ 2.1$ ) is 0 . This means that $M$ is an $l^{\prime}$-real parameter family of analytic submanifolds of complex dimension $m$, at least locally, by Frobenius' theorem, and again, the approximation theorem for these is proved in [13]. In this case it is easy to see that $M$ is not extendible. Any real linear subspace of $C^{n}$ is Levi flat. The next two are examples of a generic four real dimensional $\mathrm{CR}$ submanifold of $C^{3}$ for which $e(M)>0$.

(2.5.1). Let $M^{4} \subset C^{3}$ be given by $z_{1} \bar{z}_{1}+z_{2} \bar{z}_{2}+z_{3} \bar{z}_{3}=1 ; z_{1}-\bar{z}_{1}=0 ; z_{1} \neq 1, z_{1} \neq-1$. A basis for the sections of $H\left(M^{4}\right)$ can be given by $\zeta$ and $\zeta$, where $\zeta=\bar{z}_{3}\left(\partial / \partial z_{2}\right)$ $-\bar{z}_{2}\left(\partial / \partial z_{3}\right)$. Calculating the bracket we get

$$
\eta=[\zeta, \zeta]=z_{2} \frac{\partial}{\partial z_{2}}+z_{3} \frac{\partial}{\partial z_{3}}-\bar{z}_{2} \frac{\partial}{\partial \bar{z}_{2}}-\bar{z}_{3} \frac{\partial}{\partial \bar{z}_{3}} \text {. }
$$

Thus we see that $\eta(x) \neq 0$ for $x \in M^{4}$. Taking brackets again, we get $\eta^{\prime}=[\zeta, \eta]=2 \zeta$. Thus, $e\left(M^{4}\right)=1$. Furthermore, if $V$ is an open subset of $M^{4}$ and $f$ is a CR function on $V, f$ can be extended to a CR function on some open subset of the real hyperplane $z_{1}-\bar{z}_{1}=0$. 
(2.5.2). Define $M^{4} \subset C^{3}$ by $z_{1} \bar{z}_{1}-z_{2} \bar{z}_{2}=1 ; z_{3} \bar{z}_{3}-z_{2} \bar{z}_{2}=2$. It is easy to see that $M^{4}$ is a generic CR submanifold of $C^{3}$. On $M^{4} \cap\left\{z_{1} \neq 0, z_{3} \neq 0\right\}$, the vector fields $\zeta$ and $\zeta$, where

$$
\zeta=\frac{\bar{z}_{2}}{\bar{z}_{1}} \frac{\partial}{\partial z_{1}}+\frac{\partial}{\partial z_{2}}+\frac{\bar{z}_{2}}{\bar{z}_{3}} \frac{\partial}{\partial z_{3}}
$$

form a basis for the sections of $H\left(M^{4}\right)$. A calculation shows that the vector fields $\zeta, \zeta, \eta=[\zeta, \zeta]$, and $\eta^{\prime}=[\zeta, \eta]$ are linearly independent when $z_{2} \neq 0$. For any open subset $V$ of $M^{4} \cap\left\{z_{1} \neq 0, z_{2} \neq 0, z_{3} \neq 0\right\}$ we therefore have that $e(V)=2$. In this situation, any $\mathrm{CR}$ function on $V$ can be extended to a holomorphic function on some open subset of $C^{3}$ whose boundary contains $V$. This example is similar to H. Lewy's [10].

\section{The Cauchy problem for the $\bar{\partial}$ operator.}

3.1. The $L^{2}$ solution. Let $\rho$ be a real valued $C^{\infty}$ function defined on some open set $D$ of $C^{n}$, with $\operatorname{grad} \rho \neq 0$ and let $z_{0} \in D$ be such that $\rho\left(z_{0}\right)=0$. Suppose that the Levi form of $\rho$ has $r, 1 \leqq r \leqq n$, positive eigenvalues at $z_{0}$. Then by means of a holomorphic change of coordinates we can assume that $z_{0}$ is the origin, and that $\rho$ is of the form

$$
\begin{aligned}
\rho(z)= & z_{1}+\bar{z}_{1}+a_{11}\left|z_{1}\right|^{2}+2 \operatorname{Re}\left(z_{1} \sum_{j=2}^{n} a_{1 j} \bar{z}_{j}\right) \\
& +\sum_{j=2}^{r} \lambda_{j}\left|z_{j}\right|^{2}+\sum_{j=r+1}^{n} \nu_{j}\left|z_{j}\right|^{2}+\sigma(z),
\end{aligned}
$$

where $z=\left(z_{1}, \ldots, z_{n}\right), \sigma(z)=O\left(|z|^{3}\right)$ as $|z| \rightarrow 0, \lambda_{j}>0$ for $j=2, \ldots, r$, and the matrix

$$
\left|\begin{array}{cccc}
a_{11} & a_{12} & \cdots & a_{1 r} \\
a_{12} & \lambda_{2} & \cdots & 0 \\
\vdots & \vdots & & \vdots \\
a_{1 r} & 0 & \cdots & \lambda_{r}
\end{array}\right|
$$

is positive definite. Now consider the function

$$
\tilde{\rho}(z)=z_{1}+\bar{z}_{1}-\beta \sum_{j=r+1}^{n}\left|z_{j}\right|^{2} \quad(\beta>0) .
$$

Let $\Omega$ be an open connected neighborhood of 0 in $C^{n}$ and define for $\varepsilon, \eta \geqq 0$ the sets

$$
W(\varepsilon, \eta, \rho)=\{z \in \Omega ; \rho(z)<\varepsilon, \tilde{\rho}(z)>-\eta\} .
$$

We claim that taking $\Omega$ small enough and $\beta>0$ big enough, then

$$
\operatorname{diam} W(\varepsilon, \eta, \rho) \rightarrow 0 \quad \text { as } \varepsilon^{2}+\eta^{2} \rightarrow 0
$$


In fact, $z \in W(\varepsilon, \eta, \rho)$ implies that

$$
\begin{aligned}
\varepsilon> & z_{1}+\bar{z}_{1}-\max \left(\left|\nu_{j}\right| ; j=r+1, \ldots, n\right) \cdot \sum_{r+1}^{n}\left|z_{j}\right|^{2}+a_{11}\left|z_{1}\right|^{2} \\
& +2 \operatorname{Re}\left(z_{1} \sum_{j=2}^{n} a_{1 j} \bar{z}_{j}\right)+\sum_{j=2}^{r} \lambda_{j}\left|z_{j}\right|^{2}+\sigma \\
\geqq & -\eta+\left(\beta-\max \left|\nu_{j}\right|\right) \sum_{j=r+1}^{n}\left|z_{j}\right|^{2} \\
& +a_{11}\left|z_{1}\right|^{2}+2 \operatorname{Re}\left(z_{1} \sum_{j=2}^{n} a_{1 j} \bar{z}_{j}\right)+\sum_{j=2}^{r} \lambda_{j}\left|z_{j}\right|^{2}+\sigma .
\end{aligned}
$$

Choosing $\Omega$ small enough, we can make $\sup _{\Omega}|\sigma| \leqq \delta \sum_{j=1}^{n}\left|z_{j}\right|^{2}$ for any given $\delta>0$. Observe also that if $B$ is a hermitian positive definite $r \times r$ matrix and $\mathscr{C}$ is any $r \times(n-r)$ matrix, then we can choose $\mu>0$ big enough so that the $n \times n$ matrix

$$
A=\left(\begin{array}{cc}
B & \mathscr{C} \\
t \overline{\mathscr{C}} & \mu \mathrm{Id}
\end{array}\right)
$$

is positive definite. To see this, consider $\boldsymbol{R}^{n}=\boldsymbol{R}^{r} \oplus \boldsymbol{R}^{n-r}$ and write

$$
\left({ }^{t} \bar{a},{ }^{t} \bar{b}\right) \cdot A \cdot\left(\begin{array}{l}
a \\
b
\end{array}\right)={ }^{t} \bar{a} B a+{ }^{t} \bar{a} \mathscr{C} b+{ }^{t} \bar{b}^{t} \overline{\mathscr{C}} a+{ }^{t} \bar{b} \mu \text { Id } b .
$$

Now there is a constant $c$ such that

$$
\left|{ }^{t} \bar{a} \mathscr{C} b+{ }^{t} \bar{b}^{t} \overline{\mathscr{C}} a\right| \leqq c\|b\|\|a\|,
$$

where $\|b\|^{2}={ }^{t} \bar{b} \cdot b$ and $\|a\|^{2}={ }^{t} \bar{a} \cdot a$, and there is a constant $c_{1}>0$ such that ${ }^{t} \bar{a} B a$ $\geqq c_{1}\|a\|^{2}$ and ${ }^{t} \bar{b} \mu$ Id $b \geqq \mu\|b\|^{2}$. From (3.1.5) we can make

$$
\left|{ }^{t} \bar{a} \mathscr{C} b+{ }^{t} \bar{b}^{t} \overline{\mathscr{C}} a\right| \leqq\left(c_{1} / 2\right)\|a\|^{2}+c_{2}\|b\|^{2},
$$

for some $c_{2}$ big enough. It is therefore enough to take $\mu \geqq 2 c_{2}$ to make $A$ positive definite. Putting these remarks together, we have that if $\beta$ is big enough, there is a constant $c>0$ such that

$$
c \sum_{j=1}^{n}\left|z_{j}\right|^{2} \leqq \varepsilon+\eta \quad \text { for } z \in W(\varepsilon, \eta, \rho)
$$

which proves (3.1.4).

We fix $\beta$ and $\Omega$ so that (3.1.4) holds, and so that in addition, the gradient of $\rho$ does not vanish on $\Omega$.

It is important to observe that the Levi form of $\tilde{\rho}$ has everywhere $r$ eigenvalues equal to 0 , and that for $\varepsilon$ and $\eta$ small enough there is a Stein open set $U$ with

$$
\{z \in U ; \rho(z)<\varepsilon\}=W(\varepsilon, \eta, \rho) .
$$

This is a consequence of the fact that for $\varepsilon, \eta$ small enough, the boundary of $W(\varepsilon, \eta, \rho)$ consists of the two pieces $\rho=\varepsilon$ and $\tilde{\rho}=-\eta$, as one sees from (3.1.4). 
Definition 3.1.1. Given two open subsets $D \subset E$ of a complex manifold, we say that $(D, E)$ is an $r$-Runge pair if for every compact subset $K$ of $D$ we can find a real valued function $\phi \in C^{\infty}(E)$ such that

(i) for $\alpha<\sup _{E} \phi,\{z \in E ; \phi(z)<\alpha\}$ is relatively compact in $E$;

(ii) the Levi form of $\phi$ has at least $r$ positive eigenvalues at every point of $E$;

(iii) $\hat{K}=\left\{z \in E ; \phi(z) \leqq \sup _{K} \phi\right\} \subset \subset D$.

Now consider the sets

$$
T\left(\varepsilon, \delta_{1}, \delta_{2}\right)=\left\{z \in \Omega ; \rho(z)<\varepsilon,-\delta_{2}<\tilde{\rho}(z)<-\delta_{1}\right\}
$$

for $\varepsilon, \delta_{1}, \delta_{2}>0$ small enough, and $\delta_{1}<\delta_{2}$. We have

$$
T\left(\varepsilon, \delta_{1}, \delta_{2}\right)=W\left(\varepsilon, \delta_{2}, \rho\right)-\bar{W}\left(\varepsilon, \delta_{1}, \rho\right) .
$$

LEMMA 3.1.2. For $\varepsilon, \eta>0$ small enough, there is a sequence $\left\{E_{i}\right\}$ of domains with $a C^{\infty}$ boundary and $E_{i} \subset E_{i+1}, \bigcup_{i} E_{i}=W(\varepsilon, \eta, \rho)$ and such that if $0<\varepsilon_{1} \leqq \varepsilon, \delta_{1}<\delta_{2}$ $\leqq \eta$, the pair $\left(T\left(\varepsilon_{1}, \delta_{1}, \delta_{2}\right) \cap E_{i}, E_{i}\right)$ is $r$-Runge for every $i$.

Proof. Let $K \subset \subset T\left(\varepsilon_{1}, \delta_{1}, \delta_{2}\right)$. There are positive numbers $\varepsilon^{\prime}<\varepsilon_{1}, \delta_{1}^{\prime}>\delta_{1}, \delta_{2}^{\prime}<\delta_{2}$ with $\delta_{1}^{\prime}<\delta_{2}^{\prime}$ such that $K \subset \subset T\left(\varepsilon^{\prime}, \delta_{1}^{\prime}, \delta_{2}^{\prime}\right)$. Let $\tau(z)=\sum_{1}^{n}\left|z_{i}\right|^{2}$ and let $a>0$ be small enough so that on $K$ we have

$$
\begin{aligned}
& \tilde{\rho}(z)+a \tau(z)<-\delta_{1}^{\prime}, \\
& -\log \left[(\tilde{\rho}+\eta)\left(\eta-\delta_{2}^{\prime}\right)^{-1}\right]+a \tau(z)<0 .
\end{aligned}
$$

Write

$$
\begin{aligned}
& \phi_{1}(z)=-\log \left[(\varepsilon-\rho(z))\left(\varepsilon-\varepsilon^{\prime}\right)^{-1}\right], \\
& \phi_{2}(z)=\tilde{\rho}(z)+a \tau(z)+\delta_{1}^{\prime}, \\
& \phi_{3}(z)=-\log \left[(\tilde{\rho}+\eta)\left(\eta-\delta_{2}^{\prime}\right)^{-1}\right]+a \tau(z) .
\end{aligned}
$$

Then $\phi_{1}, \phi_{2}$ and $\phi_{3}$ belong to $C^{\infty}(W(\varepsilon, \eta, \rho))$ and are such that their Levi form has everywhere in $W(\varepsilon, \eta, \rho) r$ positive eigenvalues (corresponding to the variables $\left.z_{1}, \ldots, z_{r}\right)$. Therefore the function $\phi_{0}=\max \left(\phi_{1}, \phi_{2}, \phi_{3}\right)$ has, everywhere on $W(\varepsilon, \eta), r$ positive eigenvalues in the distribution sense. We also have that $\phi_{0}(z)$ tends to $+\infty$ when $z$ tends to a point in the boundary of $W(\varepsilon, \eta)$, which as we have taken $\varepsilon$ and $\eta$ small enough, consists by virtue of (3.1.4) of the two pieces $\{\rho=\varepsilon\}$ and $\{\tilde{\rho}=-\eta\}$. On the other hand, by (3.1.8) and (3.1.9),

$$
K \subset\left\{\phi_{0}<0\right\} \text { and } \phi_{0}>0 \text { on boundary of } T\left(\varepsilon_{1}, \delta_{1}, \delta_{2}\right) .
$$

Hence $\phi_{0}$ satisfies all the requirements in Definition 3.1.1 for the pair $\left(T\left(\varepsilon_{1}, \delta_{1}, \delta_{2}\right), W(\varepsilon, \eta, \rho)\right)$, except that it does not belong to $C^{\infty}(W(\varepsilon, \eta, \rho))$. To complete the proof, we take a slightly smaller set $W_{0} \subset \subset W(\varepsilon, \eta, \rho)$, and set

$$
0<\alpha_{0}=\inf \left\{d(z, \partial W(\varepsilon, \eta, \rho)), z \in W_{0}\right\} .
$$


Then take a function $\chi$ of $\tau(z)^{1 / 2}=|z|$ such that $\chi \in C^{\infty}\left(C^{n}\right), \chi \geqq 0$ and $\chi(|z|)=0$ for $|z| \geqq 1$, normalized so that $\int \chi d V=1$. For $0<\alpha<\alpha_{0}$ we take

$$
\phi_{\alpha}(z)=\int \phi_{0}\left(z-\alpha z^{\prime}\right) \chi\left(z^{\prime}\right) d V\left(z^{\prime}\right) \text {. }
$$

Then $\phi_{\alpha} \in C^{\infty}\left(W_{0}\right)$, and its Levi form has everywhere $r$ positive eigenvalues on $W_{0}$. Furthermore $\phi_{\alpha} \searrow \phi_{0}$ as $\alpha \searrow 0$. The domains $E_{i}$ are then chosen so that $E_{i}=\left\{\phi_{\alpha}<M_{i}\right\}$, and by Morse theory we can choose them so that their boundary is smooth. By letting $W_{0}$ approach $W(\varepsilon, \eta, \rho)$ and $M_{i} \rightarrow \infty$, we construct the family $\left\{E_{i}\right\}$ with the desired properties. Q.E.D.

The reason for considering Runge pairs is the following: let $D \subset E$ as before and let $H_{c}^{p, q}(D)$ stand for the $\bar{\partial}$-cohomology space of type $(p, q)$ with compact support, obtained by taking the quotient $\operatorname{Ker} \bar{\partial}: \mathscr{A}_{0}^{(p, q)}(D) \rightarrow \mathscr{A}_{0}^{(p, q+1)}(D)$ over $\bar{\partial}\left(\mathscr{A}_{0}^{p, q-1}(D)\right)$, where $\mathscr{A}_{0}^{(p, q)}$ is the space of forms of type $(p, q)$ whose coefficients are $C^{\infty}$ and compactly supported in $D$. Then there is a natural mapping $H_{c}^{p, q}(D) \rightarrow H_{c}^{p, q}(E)$, induced by the natural inclusion $\mathscr{A}_{0}^{p, q}(D) \rightarrow \mathscr{A}_{0}^{p, q}(E)$. For Runge pairs more can be said:

THEOREM 3.1.3. If $(D, E)$ is an $r$-Runge pair, the natural mapping $H_{c}^{p, q}(D)$ $\rightarrow H_{c}^{p, q}(E)$ is injective for $q \leqq r$. Therefore if $f \in L_{p, q}^{2}(D)$ (the space of $(p, q)$ forms on $D$ with $L^{2}$ coefficients), and $f$ has compact support in $D$, and $f=\vec{\partial} g$ for $g \in L_{p, q-1}^{2}(E)$ with $g$ compactly supported in $E$, we can find $h \in L_{p, q-1}^{2}(D)$ compactly supported in $D$ so that $f=\bar{\partial} h$, if $q \leqq r$. Furthermore $h$ can be chosen such that $\|h\|$ $\leqq C\|f\|$, where \|\| stands for the $L^{2}$ norm and the constant $C$ does not depend on $f$.

For the proof of this result we refer to Andreotti and Vesentini [2, Lemma 29, p. 122], where the above $L^{2}$ estimate does not appear in the statement but is obvious from the proof. One can also obtain it from Hörmander [6] and the use of Serre duality. See also Andreotti and Grauert [1].

As a consequence of Lemma 3.1.2, we get

COROLlaRY 3.1.4. The conclusions of Theorem 3.1.3 hold for $D=T\left(\varepsilon_{1}, \delta_{1}, \delta_{2}\right)$ and $E=W(\varepsilon, \eta, \rho)$ for $\varepsilon, \eta>0$ small enough and $\varepsilon_{1} \leqq \varepsilon, \delta_{1}<\delta_{2} \leqq \eta$.

We will also need the following result, whose proof is carried out along the same lines as that of Theorem 3.1.3. (See Andreotti and Vesentini [2, Theorem 5, p. 111], or again, Hörmander [6] and Serre duality.)

THEOREM 3.1.5. Let $E$ be an open set in $C^{n}$ and suppose $\phi \in C^{\infty}(E)$ satisfies (i) and (ii) of Definition 3.1.1. Then $H_{c}^{p, q}(E)=0$ for $q<r$. Furthermore, if $f$ is $a(p, q)$ form in $L_{p, q}^{2}(E)$ with compact support in $E, q<r$, and $\bar{\partial} f=0$, we can find $a(p, q-1)$ form $g$ in $L_{p, q-1}^{2}(E)$ with compact support such that $\bar{\partial} g=f$ and $\|g\| \leqq C\|f\|$, with $C$ independent of $f$.

We can now prove the main result of this number.

THEOREM 3.1.6. Let $\rho$ be a real valued $C^{\infty}$ function on some open set $\Omega$ of $C^{n}$, let $z_{0} \in \Omega$ be such that $\rho\left(z_{0}\right)=0$ and $\operatorname{grad} \rho\left(z_{0}\right) \neq 0$. Suppose that the Levi form of $\rho$ 
has $r$ positive values at $z_{0}$. Then there is $\varepsilon_{0}>0$ and $\eta>0$ with the following property:

Let $0 \leqq \varepsilon<\varepsilon_{0}$, and let $U$ be an open neighborhood of $z_{0}$ with $U \cap\{z \in \Omega ; \rho(z)<\varepsilon\}$ $=W(\varepsilon, \eta, \rho)$. Also let $V$ be an open set with $\bar{U} \subset V$, and call $d(U, V)=$ distance between $\bar{U}$ and $C^{n}-V$. Then given a form $f \in L_{p, q}^{2}(V)$ with $0<q<r$ such that $\bar{\partial} f=0$ on $V$ and $f \equiv 0$ on $V \cap\{z \in \Omega ; \rho(z)>\varepsilon\}$, we can find a form $g \in L_{p, q-1}^{2}(U)$ such that $\bar{\partial} g=f$ on $U$ and $g \equiv 0$ on $U \cap\{z \in \Omega ; \rho(z)>\varepsilon\}$. Furthermore, $g$ can be chosen so that $\|g\| \leqq C\|f\|$, where $C$ only depends on $d(U, V)$, and $C=O\left(d(U, V)^{-1}\right)$.

Proof. Let $\eta^{\prime \prime \prime}>\eta^{\prime \prime}>\eta^{\prime}>\eta$ be such that $W\left(\varepsilon, \eta^{\prime \prime \prime}\right) \subset V$, and such that Corollary 3.1.4 holds for $0 \leqq \varepsilon<\varepsilon_{0}$ and for $\eta^{\prime \prime}$. Let $\chi(t) \in C^{\infty}(\boldsymbol{R})$ be such that $0 \leqq \chi \leqq 1, \chi(t)=1$ for $t \geqq-\eta^{\prime}$ and $\chi(t)=0$ for $t \leqq-\eta^{\prime \prime}$. Then if $\varepsilon<\varepsilon^{\prime}<\varepsilon_{0}$, the form $\chi(\tilde{\rho}) \cdot f$ will have compact support in $W\left(\varepsilon^{\prime}, \eta^{\prime \prime \prime}, \rho\right)$ (if we have chosen $\varepsilon_{0}$ and $\eta$ small enough to begin with) by (3.1.4). Observe now that $\bar{\partial}(\chi(\tilde{\rho}) \cdot f)=\bar{\partial} \chi(\tilde{\rho}) \wedge f$ is a $(p, q+1)$ form with compact support in $T\left(\varepsilon^{\prime}, \eta, \eta^{\prime \prime}\right)$. As $q+1 \leqq r$, we can therefore find a $(p, q)$ form $\omega$ compactly supported in $T\left(\varepsilon^{\prime}, \eta, \eta^{\prime \prime \prime}\right)$ such that $\bar{\partial} \omega=\bar{\partial}(\chi(\tilde{\rho}) \cdot f)$, with

$$
\|\omega\| \leqq C_{1}\|f\|
$$

where $C_{1}$ depends only on the first derivative of $\chi$. Then the $(p, q)$ form $\chi(\tilde{\rho}) \cdot f-\omega$ $=h$ is $\bar{\partial}$-closed on $V$ and its support is compact in $W\left(\varepsilon^{\prime}, \eta^{\prime \prime \prime}, \rho\right)$. Also, it coincides with $f$ on $W\left(\varepsilon^{\prime}, \eta, \rho\right)$. By Theorem 3.1 .5 we can find a $(p, q-1)$ form $g_{\varepsilon^{\prime}}$ such that $\bar{\partial} g_{\varepsilon^{\prime}}=h$ on $W\left(\varepsilon^{\prime}, \eta^{\prime \prime \prime}, \rho\right)$ and supp $g_{\varepsilon^{\prime}} \subset \subset W\left(\varepsilon^{\prime}, \eta^{\prime \prime \prime}, \rho\right)$. Furthermore by (3.1.12), $\left\|g_{\varepsilon^{\prime}}\right\| \leqq C\|f\|$, where $C$ depends only on $C_{1}$. (It can be seen in [2] that it does not depend on $\varepsilon^{\prime}, \varepsilon<\varepsilon^{\prime}<\varepsilon_{0}$.) This last inequality implies that by weak compactness of the unit ball in $L^{2}$, there is a weakly convergent sequence $g_{\varepsilon^{\prime}}$ with $\varepsilon^{\prime} \searrow \varepsilon$. If we call $g$ its weak limit, we easily verify that $\bar{\partial} g=f$ on $U$, that $g=0$ if $\rho>\varepsilon$, and that $\|g\| \leqq C\|f\|$. Q.E.D.

Now assume that

$$
\rho=\max \left(\rho_{1}, \ldots, \rho_{m}\right)
$$

where the Levi form of each $\rho_{i}$ has $r$ positive eigenvalues at $z_{0}$, corresponding to the same $r$-dimensional space. Then each $\rho_{i}, i=1, \ldots, m$, can be expressed on a neighborhood of $z_{0}$ in the form (3.1.1). Furthermore, assume that $d \rho_{1} \wedge \cdots \wedge d \rho_{m}\left(z_{0}\right) \neq 0$.

The following result will not be needed in the sequel, but might have some interest.

COROLlaRY 3.1.7. The conclusions of Theorem 3.1.6 hold for $\rho$ as in (3.1.13), with a set $W(\varepsilon, \eta, \rho)$ which contains $\bigcap_{i=1}^{m} W\left(\varepsilon, \eta, \rho_{i}\right)$.

Proof. We consider the case $m=2$, the general case being different only in that it requires a bigger burden of notation. Write

$$
\rho=\max \left(\rho_{1}, \rho_{2}\right)=\frac{1}{2}\left\{\rho_{1}+\rho_{2}+\left|\rho_{1}-\rho_{2}\right|\right\},
$$

and consider the Taylor expansion of the function $\left(\varepsilon^{2}+t^{2}\right)^{1 / 2}$ about $t=\frac{1}{2}$. Call $P_{\varepsilon, n}(t)$ the first $n$ terms, and set

$$
\phi_{\varepsilon, n}=\frac{1}{2}\left\{\rho_{1}+\rho_{2}+P_{\varepsilon, n}\left(\rho_{1}-\rho_{2}\right)\right\} .
$$


With $\phi_{n}=\phi_{1 / n, 2 n+1}$ it is not hard to see that there is a neighborhood $\Omega_{0}$ of $z_{0}$ in $C^{n}$ such that $\phi_{n} \in C^{\infty}\left(\Omega_{0}\right)$ for each $n, \phi_{n} \searrow \rho$ as $n \rightarrow \infty$ on $\Omega_{0}$, and the Levi form of $\phi_{n}$ has at least $r$ positive eigenvalues on $\Omega_{0}$, for every $n$, which are bounded from below by a positive constant $C$ independent of $n$. Furthermore, recalling (3.1.2), we have, for every $n, \tilde{\phi}_{n}=\frac{1}{2}\left(\rho_{1}+\rho_{2}\right)^{\sim}$, which proves that if $\varepsilon^{\prime}>\varepsilon$ and $n$ is big enough, then for any $\eta>0, W\left(\varepsilon^{\prime}, \eta, \phi_{n}\right) \supset W\left(\varepsilon, \eta, \rho_{1}\right) \cap W\left(\varepsilon, \eta, \rho_{2}\right)$. The situation being as in the statement of Theorem 3.1.6, the proof of the corollary is completed by taking $\varepsilon^{\prime}>\varepsilon$, and applying Theorem 3.1.6 to $\phi_{n} \leqq \varepsilon^{\prime}$ for $n$ big enough, then letting $\varepsilon^{\prime} \rightarrow \varepsilon$ and taking a weak limit of $L^{2}$ solutions. Observe that we can take

$$
W(\varepsilon, \eta, \rho)=\left\{z \in \Omega_{0} ; \rho(z)<\varepsilon, \frac{1}{2}\left(\rho_{1}+\rho_{2}\right)^{\sim}>-\eta\right\} . \quad \text { Q.E.D. }
$$

Although we will not need it, it is worth noting that if $f$ in Theorem 3.1.6 belongs to $C^{\infty}(U)$, then there is a solution $g$ in $C^{\infty}(U)$. This can be proved by using the regularity results of J. J. Kohn and L. Nirenberg [Noncoercive boundary value problems, Comm. Pure Appl. Math. 18 (1965)] and it can be used to prove the $\mathrm{H}$. Lewy extension phenomenon on real hypersurfaces for cohomology classes of type $(p, q)$ with $q<n-1$.

\section{Function algebras on CR submanifolds.}

4.1. Uniform approximation with holomorphic functions. Let $K$ be a compact subset of a complex manifold $X$, and let $\mathcal{O}$ be the sheaf of germs of holomorphic functions on $X$. Let $C(K)$ stand for the uniform algebra of complex valued continuous functions on $K$, and $\Gamma(K, \mathcal{O})$ for the algebra of sections of $\mathcal{O}$ over $K$. Then there is a natural restriction mapping $\Gamma(K, \mathcal{O}) \stackrel{r}{\longrightarrow} C(K)$, and we call $A(K)=$ closure of $r(\Gamma(K, \mathcal{O}))$ in $C(K)$. Similarly, if $M$ is a CR submanifold of $C^{n}$ and $K$ is a compact subset of $M, \mathcal{O}_{M}$ denotes the sheaf of germs of $\mathrm{CR}$ functions on $M$ (solutions of $\left.\xi_{i}(f)=0\right)$ and $A_{M}(K)$ is the closure of $r\left(\Gamma\left(K, \mathcal{O}_{M}\right)\right)$ in $C(K)$.

The following result is proved in [13]: Let $K$ be a compact subset of $M$, where $M$ is a totally real submanifold of a complex manifold $X$ (i.e. $\mathrm{CR} \operatorname{dim} M=0$ ). Then $A(K)=C(K)$.

When $M$ is not totally real, the best we can expect is of course $A(K)=A_{M}(K)$. Our aim is to establish this result locally. This was done in [13] in two cases: $M$ a real smooth hypersurface ( $\left.\operatorname{codim}_{R} M=1\right)$, and $M$ Levi flat (any dimension).

Let $M$ be a CR submanifold of $C^{n}, z_{0} \in M$, and suppose that at least one of the following conditions is satisfied.

(I) There is a smooth real hypersurface containing $M$ whose Levi form restricted to $H_{z_{0}}(M)$ has at least one positive and at least one negative eigenvalue.

(II) There is a smooth real hypersurface containing $M$ whose Levi form restricted to $H_{z_{0}}(M)$ has all its eigenvalues of the same sign $\neq 0$. 
THeOREM 4.1.1. Let $M$ be a $C R$ submanifold of $C^{n}$ and let $z_{0} \in M$. Assume that either (I) or (II) of (4.1.1) holds. Then there is a fundamental system of compact neighborhoods $\{K\}$ of $z_{0}$ in $M$ such that if $K \in\{K\}$ then $A(K)=A_{M}(K)$.

We will use the following

LEMMA 4.1.2. Let $\left\{\rho_{1}, \ldots, \rho_{k}\right\}$ and $\left\{\rho_{1}^{\prime}, \ldots, \rho_{k}^{\prime}\right\}$ be two defining systems for $M$, defined on some neighborhood $D$ of $M$, with $\rho_{i}^{\prime}=\sum_{j=1}^{k} a_{i j} \rho_{j}$. Assume that the $C^{\infty}$ real $k \times k$ matrix $\left(a_{i j}\right)$ restricted to $M$ is a symmetric positive definite matrix. For $\varepsilon>0$ define

$$
T(\varepsilon)=\left\{z \in D ; \rho_{i}(z)<\varepsilon, \rho_{i}^{\prime}(z)>-\varepsilon ; i=1, \ldots, k\right\} .
$$

Then if $D$ is taken small enough, $\bigcap_{\varepsilon>0} T(\varepsilon)=M$.

Proof. $z \in T(\varepsilon)$ means that $\varepsilon-\rho_{i}(z)>0, i=1, \ldots, k$, and $\sum_{j=1}^{k} a_{i j} \rho_{j}(z)+\varepsilon>0$, $i=1, \ldots, k$. Therefore if $D$ is small enough, $z \in T(\varepsilon)$ implies that

$$
\sum_{i=1}^{k} \rho_{i}^{2}(z) \leqq c \sum_{i, j} a_{i j}(z) \rho_{i} \rho_{j}(z)<\varepsilon c\left(k \sum_{j=1}^{k} a_{i j}(z) \rho_{j}(z)-\sum_{i=1}^{k} \rho_{i}(z)+k \varepsilon\right)
$$

for some constant $c$, and therefore, $\sum_{i=1}^{k} \rho_{i}^{2}(z)$ tends to 0 as $\varepsilon \rightarrow 0$. Q.E.D.

Proof of Theorem 4.1.1. Obviously it is enough to prove that any $f$ in $\Gamma\left(K, \mathcal{O}_{M}\right)$ can be uniformly approximated on $K$ by elements in $\Gamma(K, \mathcal{O})$.

We set $k=\operatorname{codim}_{R} M, l=\mathrm{CR} \operatorname{codim} M, m=\mathrm{CR} \operatorname{dim} M$.

Consider first case (I). Using Lemma 2.4.4, we can find two CR regular defining systems for $M$ on some neighborhood $D$ of $z_{0},\left\{\rho_{1}, \ldots, \rho_{k}\right\}$ and $\left\{\rho_{1}^{\prime}, \ldots, \rho_{k}^{\prime}\right\}$ such that

(i) $\bar{\partial} \rho_{1}\left(z_{0}\right) \wedge \cdots \wedge \bar{\partial} \rho_{l} \neq 0 ; \bar{\partial} \rho_{1}^{\prime}\left(z_{0}\right) \wedge \cdots \wedge \bar{\partial} \rho_{l}^{\prime} \neq 0$ on $D$.

(ii) The Levi form of $\rho_{j}$ has at least $l+1$ positive eigenvalues on $D$ for $j=1, \ldots, k$, and the Levi form of $\rho_{j}^{\prime}$ on $D$ has at least $l+1$ negative eigenvalues for $j=1, \ldots, k$.

(iii) $\rho_{i}^{\prime}=\sum_{1}^{k} a_{i j} \rho_{j}$ on $D$, where the $C^{\infty}$ real matrix $\left(a_{i j}\right)$ is equal to the identity on $M \cap D$.

Let $U \subset D$ be an open neighborhood of $z_{0}$ in $C^{n}$ with $U \cap M=V$ for which the assertions of Lemma 2.4.2 and Corollary 2.4.3 hold. Then if $f$ is a CR function on $V$, it has an extension $\hat{f}$ to $U$ satisfying (2.4.3), and the order $r$ of vanishing of $\bar{\partial} \hat{f}$ on $M$ will be chosen at the end of the proof. (Or it can be made $\infty$ from the beginning.)

Consider now a family of functions $\psi_{t}(x)$ of a real variable $x$ in $C^{\infty}(\boldsymbol{R})$ with $t \in R, t>0$, such that

(1) $\psi_{t}(x)=1$ for $x \geqq-t$,

(2) $\psi_{t}(x)=0$ for $x \leqq-2 t$,

(3) $0 \leqq \psi_{t}(x) \leqq 1$ for every $x$ and $t$,

(4) $\left|d^{m} \psi_{t}(x) / d x^{m}\right| \leqq C t^{-m}$ for some constant $C$ and every $m=1,2, \ldots$ 
Let

$$
\psi_{t, i}=\psi_{t}\left(\rho_{i}\right) \cdot \psi_{t}\left(-\rho_{i}^{\prime}\right), \quad i=1, \ldots, l .
$$

We use the following notation: for a multi-index $J$ of length $\|J\|=N$, and differential forms $f_{j_{1}}, \ldots, f_{j_{N}}$, we write

$$
\bigwedge_{J} f_{j}=\bigwedge_{j \in J} f_{j}=f_{j_{1}} \wedge \cdots \wedge f_{j_{N}} ; \quad \bigwedge_{J} f_{j}=1 \text { if } N=0 .
$$

We will consider multi-indices $J$ consisting of $N$ different numbers in the set $\{1, \ldots, l\}$. Let $J_{l}$ be a multi-index of length $l$; we have by (2.4.3)

$$
\left(\bigwedge_{J_{l}} \bar{\partial} \psi_{t, j}\right) \wedge \bar{\partial} \hat{f} \equiv 0 \quad \text { on } U
$$

Therefore, if $J_{l-1}$ is of length $l-1$, the $(0, l)$ forms

$$
\xi\left(t, J_{l-1}\right)=\psi_{t, i}\left(\bigwedge_{J_{l-1}} \bar{\partial} \psi_{t, j}\right) \wedge \bar{\partial} \hat{f} ; \quad i \notin J_{l-1} ; \quad i=1, \ldots, l,
$$

are $\bar{\partial}$-closed on $U$.

At this point we make the observation that we can assume $l<n$, for if $l=n, M$ is totally real and the theorem is proved in [13].

We now introduce the following sets: for a real number $s$ and a multi-index $J$ define

$$
R_{J}(s)=\bigcap_{j \in J}\left(\left\{z \in U ; \rho_{j}(z) \leqq-s\right\} \cup\left\{z \in U ; \rho_{j}^{\prime}(z) \geqq s\right\}\right) ;
$$

we also put $R_{J}(s)=U$ if $\|J\|=0$ and $R_{J}(s)=R_{j}(s)$ if $J=\{j\}$. From (4.1.3), (4.1.4) and (4.1.6), we see that

$$
\operatorname{supp} \xi\left(t, J_{l-1}\right) \subset R_{J_{l-1}}(t) .
$$

By (4.1.2)(iii), for any $j \in J$ the corresponding two sets in the union in (4.1.7) are disjoint. By shrinking $U$ if necessary, we can assume that $U$ is a Stein open set, and that using (3.1.6) and Theorem 3.1.6, if $t$ is small enough, then for any $j \in J_{l-1}$ we can find a form $\omega\left(t, J_{l-1}, j\right)$ of type $(0, l-1)$ in $L^{2}(U)$ such that

(1) $\bar{\partial} \omega\left(t, J_{l-1}, j\right)=\xi\left(t, J_{l-1}\right)$ on $U$

(2) $\operatorname{supp} \omega\left(t, J_{l-1}, j\right) \subset R_{j}(t)$,

(3) $\left\|\omega\left(t, J_{l-1}, j\right)\right\| \leqq C\left\|\xi\left(t, J_{l-1}\right)\right\|, C=$ absolute constant,

(4) $\omega\left(t, J_{l-1}, j\right)$ is antisymmetric in the indices of $J_{l-1}$, for any $t$ and any $j$.

This can be done if $1<l<n$ by virtue of (4.1.2)(ii).

Pick now a multi-index $J_{l-2}$ with $\left\|J_{l-2}\right\|=l-2$. We write $j J_{l-2}$ for the multiindex obtained by adjoining $j$ at the beginning of $J_{l-2}$.

For $i \in J_{l-2}$ we define the $(0, l-1)$ forms in $L^{2}(U)$

$$
\xi\left(t, J_{l-2}, i\right)=\prod_{j \notin J_{l-2}} \psi_{t, j} \cdot \bigwedge_{J_{l-2}} \bar{\partial} \psi_{t, j} \wedge \bar{\partial} \hat{f}-\sum_{j \notin J_{l-2}} \omega\left(t, j J_{l-2}, i\right) .
$$


These are $\bar{\partial}$-closed on $U$ by (1) of (4.1.9), and the support of $\xi\left(t, J_{l-2}, i\right)$ is contained in $R_{i}(t)$ by (2) of (4.1.9), and the fact that $i \in J_{l-2}$.

If now $l>2$, proceeding as before we can find forms $\omega\left(t, J_{l-2}, i\right)$ of type $(0, l-2)$ such that

(1) $\omega\left(t, J_{l-2}, i\right) \in L^{2}(U)$ and $\bar{\partial} \omega\left(t, J_{l-2}, i\right)=\xi\left(t, J_{l-2}, i\right)$ on $U$,

(2) $\operatorname{supp} \omega\left(t, J_{l-2}, i\right) \subset R_{i}(t)$,

(3) $\left\|\omega\left(t, J_{l-2}, i\right)\right\| \leqq C\left\|\xi\left(t, J_{l-2}, i\right)\right\| ; C=$ absolute constant,

(4) $\omega\left(t, J_{l-2}, i\right)$ is antisymmetric in the indices of $J_{l-2}$, for any $t$ and $i$.

(Observe that (4) of (4.1.11) is possible as a consequence of (4) of (4.1.9).) Then if $J_{l-3}$ is a multi-index of length $l-3$, and $i \in J_{l-3}$, we have (for $l>3$ )

$$
\begin{aligned}
\bar{\partial}\left[\prod_{j \notin J_{l-3}} \psi_{t, j} \cdot \bigwedge \bar{\partial} \psi_{t, j} \wedge \bar{\partial} \hat{f}\right] & =\sum_{h \notin J_{l-3}} \prod_{j \notin h J_{l-3}} \psi_{t, j} \cdot \bigwedge_{h J_{l-3}} \bar{\partial} \psi_{t, j} \wedge \bar{\partial} \hat{f} \\
& =\sum_{h \notin J_{l-3}} \xi\left(t, h J_{l-3}, i\right)+\sum_{h \notin J_{l-3}} \sum_{j \notin h J_{l-3}} \omega\left(t, j h J_{l-3}, i\right) .
\end{aligned}
$$

The double sum in the right-hand side vanishes by (4) of (4.1.9). Therefore, the $(0, l-2)$ forms

$$
\xi\left(t, J_{l-3}, i\right)=\prod_{j \notin J_{l-3}} \psi_{t, j} \cdot \bigwedge_{J_{l-3}} \bar{\partial} \psi_{t, j} \wedge \bar{\partial} \hat{f}-\sum_{j \notin J_{l-3}} \omega\left(t, j J_{l-3}, i\right)
$$

are in $L^{2}(U)$ and are $\bar{\partial}$-closed on $U$. Also, their support is contained in $R_{i}(t)$. Continuing in this way, we finally arrive at a $\bar{\partial}$-closed $(0,1)$ form in $L^{2}(U)$ :

$$
\prod_{j=1}^{l} \psi_{t, j} \cdot \bar{\partial} \hat{f}-\sum_{j=1}^{l} \omega(t, j, j)=\zeta_{t} .
$$

The forms $\zeta_{t}$ and $\bar{\partial} \hat{f}$ coincide on the set

$$
\bigcap_{j=1}^{l}\left\{z \in U ; \rho_{j}(z) \geqq-t\right\} \cap \bigcap_{j=1}^{l}\left\{z \in U ; \rho_{j}^{\prime}(z) \leqq t\right\},
$$

where $\psi_{t, j} \equiv 1$ for $j=1, \ldots, l$, and where $\omega(t, j, j)=0$ for $j=1, \ldots, l$. This set is a neighborhood of $U \cap M$ for every $t>0$. In view of Lemma 4.1.2, of (4.1.3)(2) and (4.1.3)(4), we can make the $L^{2}$ norm of the forms (4.1.6) (and of any number of its derivatives) arbitrarily small by taking $t$ small, if we choose $r$, the order of the vanishing of $\bar{\partial} \hat{f}$ on $\rho_{1}=\cdots=\rho_{l}=0$, big enough to begin with. Using (4.1.9)(3) and (4.1.11)(3), etc., the same is true of the successive forms $\xi$ and $\omega$, and therefore the $L^{2}(U)$ norm of $\zeta_{t}$ can be made arbitrarily small by taking $t$ small enough. (The same is true of any Sobolev norm.) We then solve the equation

$$
\bar{\partial} v_{t}=\zeta_{t} \text { on } U
$$


with

$$
\left\|v_{t}\right\| \leqq C\left\|\zeta_{t}\right\| \quad C=\text { absolute constant. }
$$

That this can be done is a consequence of the fact that $U$ is Stein, and of the results in Hörmander ([6] or [7]).

The function $\hat{f}-v_{t}$ is therefore holomorphic on the set

$$
\sigma(t)=\bigcap_{j=1}^{l}\left\{z \in U ; \rho_{j}(z) \geqq-t\right\} \cap \bigcap_{j=1}^{l}\left\{z \in U ; \rho_{j}^{\prime}(z) \leqq t\right\}
$$

and the difference $\left|\hat{f}-\left(\hat{f}-v_{t}\right)\right|=\left|v_{t}\right|$ can be made uniformly small on compact subsets of $M \cap U$ if we take $t$ to be small, by (4.1.13) and Sobolev's Lemma. The details of the argument are identical to those in [13].

In case (II), the proof is similar, but somewhat easier. Instead of the two systems in (4.1.2), we consider just one, $\left\{\rho_{1}, \ldots, \rho_{k}\right\}$, with $\rho_{j}$ strongly plurisubharmonic in $D$ for $j=1, \ldots, k$, and $\bar{\partial} \rho_{1} \wedge \cdots \wedge \bar{\partial} \rho_{l} \neq 0$ on $D$.

Then with $\psi_{t}(x)$ as in (4.1.3) one considers

$$
\psi_{t, i}=\psi_{t}\left(\rho_{i}\right) \cdot \psi_{t}\left(-\rho_{i}\right), \quad i=1, \ldots, l .
$$

One then proceeds as in case (I), but instead of $\omega$ as in (4.1.9) one obtains

(1) $\bar{\partial} \omega\left(t, J_{l-1}, j\right)=\xi\left(t, J_{l-1}\right)$ on $U \cap\left\{\rho_{j}(z) \leqq t, j=1, \ldots, l\right\}$,

(2) $\operatorname{supp} \omega\left(t, J_{l-1}, j\right) \subset\left\{z \in U ; \rho_{j}(z) \leqq-t\right\}$,

(3) and (4) as in (4.1.9).

Then $\xi\left(t, J_{l-2}, i\right)$ are $\bar{\partial}$-closed on $U \cap\left\{\rho_{j}(z) \leqq t, j=1, \ldots, l\right\}=\Omega_{t}$. Finally one arrives at a $(0,1)$ form $\zeta_{t}$ with the same properties as above, except that it is $\bar{\partial}_{-}$ closed and defined on $\Omega_{t}$, instead of $U$. But $\Omega_{t}$ is Stein for every $t>0$, because of the assumption on the $\rho$ 's. Therefore one can solve (4.1.12) with (4.1.13) on $\Omega_{t}$, with a constant $C$ independent of $t$, and the resulting function $\hat{f}-v_{t}$ is holomorphic on the set

$$
\sigma(t)=\bigcap_{j=1}^{l}\left\{z \in U ; \rho_{j}(z) \geqq-t\right\} \cap \bigcap_{j=1}^{l}\left\{z \in U ; \rho_{j}(z) \leqq t\right\} . \quad \text { Q.E.D. }
$$

REMARK 4.1.3. We have actually proved that for a fundamental system of neighborhoods $\{U\}$ of $z_{0}$, an element $f \in \Gamma\left(\bar{U} \cap M, \mathcal{O}_{M}\right)$ can be uniformly approximated on $\bar{U} \cap M$ by elements of $\Gamma(\bar{U} \cap \hat{M}, \mathcal{O})$, where $\hat{M}$ is defined by $\rho_{1}=\ldots$ $=\rho_{l}=0$, and is a generic CR submanifold of $C^{n}$ containing $M$ (locally near $z_{0}$ ). Here $\left\{\rho_{1}, \ldots, \rho_{k}\right\}$ is a CR regular defining system for $M$, as in (4.1.2), and $l$ is as in (4.1.2)(i). This is not surprising, it being obvious from Lemma 2.4 .2 that $f \in \Gamma\left(\bar{U} \cap M, \mathcal{O}_{M}\right)$ can always be extended to $\hat{f} \in \Gamma\left(\bar{U} \cap \hat{M}, \mathcal{O}_{\hat{M}}\right)$.

4.2. Extendibility and the $H$. Lewy phenomenon. Given a set $K$ of $C^{n}$ and a connected set $K^{\prime}$ of $\boldsymbol{C}^{n}$ with $K \subset K^{\prime}, K \neq K^{\prime}$, we say that $K$ is extendible to $K^{\prime}$ if the restriction map $\Gamma\left(K^{\prime}, \mathcal{O}\right) \rightarrow \Gamma(K, \mathcal{O})$ is onto. 
If $M$ is a generic CR submanifold of $C^{n}$ with $\operatorname{dim}_{R} M \geqq n$, and $U$ is an open subset of $M$, it is easy to see that a function which is holomorphic on some open neighborhood of $\bar{U}$ and is zero on $U$ must vanish identically. Therefore, if $K=\bar{U}$ and $K$ is extendible to a set $K^{\prime}$, we have in this case that $\Gamma\left(K^{\prime}, \mathcal{O}\right) \rightarrow \Gamma(K, \mathcal{O})$ is one-to-one and onto.

Let $K$ be a compact subset of a complex manifold $X$, and let $S(K)$ be the spectrum of the uniform algebra $A(K)$. We say that $K$ is holomorphically convex if $K=S(K)$. (Of course we always have $K \subset S(K)$ by means of the evaluation map.) We say that a set $M \subset X$ is locally holomorphically convex at $z_{0} \in M$ if there is a fundamental system of compact neighborhoods $\{K\}$ of $z_{0}$ in $M$ such that each $K$ is holomorphically convex.

Greenfield [4] has proved the following theorem, making use of the techniques first introduced by Bishop [3]:

THEOREM 4.2.1. If $M$ is a generic $C R$ submanifold of $C^{n}$ with $\operatorname{dim}_{R} M \geqq n$, then $M$ is extendible to a set containing a CR submanifold $N$ with $\operatorname{dim}_{R} N=\operatorname{dim}_{R} M+e$ ( $e$ defined as in $\S 2$ ). If $e=0$, then $M$ is locally holomorphically convex.

In [5] and [16] this theorem is proved for real-analytic submanifolds of $\boldsymbol{C}^{n}$ which are not necessarily generic, and it is also proved for $C^{\infty} \mathrm{CR}$ submanifolds with $\mathrm{CR}$ codim $=1$. In these cases, one also has that $M$ is not locally extendible to a set of dimension greater than $\operatorname{dim} M+e$.

If $M$ is a CR submanifold of $C^{n}, K \subset M$ and $K^{\prime}$ is a connected set with $K^{\prime} \supset K$, $K \neq K^{\prime}$, we say that $K$ is $C R$ extendible to $K^{\prime}$ if the restriction map $A\left(K^{\prime}\right) \rightarrow \Gamma\left(K, \mathcal{O}_{M}\right)$ is onto. We then have

THEOREM 4.2.2. Let $M$ be a CR submanifold of $C^{n}, z_{0} \in M$, and suppose $M$ satisfies either one of conditions (I) or (II) of (4.1.1). Then there is a fundamental system of compact neighborhoods of $z_{0}$ in $M,\{K\}$, such that each $K \in\{K\}$ is CR extendible to a corresponding set $K^{\prime}$ which contains a CR submanifold of dimension $\operatorname{dim} M+e+k-l$.

Proof. Let $K=M \cap U$, where $U \in\{U\}$, the fundamental system of open neighborhoods of $z_{0}$ in Theorem 4.1.1. Then by Theorem 4.1.1 and Remark 4.1.3, given $f \in \Gamma\left(K, \mathcal{O}_{M}\right)$, we can first extend $f$ to $\hat{f} \in \Gamma\left(\hat{K}, \mathcal{O}_{\hat{M}}\right)$, where $\hat{K}=U \cap \hat{M}$, and then find a sequence $\left\{\hat{f}_{j}\right\} \subset \Gamma(\hat{K}, \mathcal{O})$ which converges uniformly to $\hat{f}$ on $\hat{K}$. Because $\hat{M}$ is generic and $\operatorname{dim}_{R} \hat{M} \geqq n$, we can apply Theorem 4.2.1 to find for each $\hat{f}_{j}$ an extension $g_{j} \in \Gamma\left(K^{\prime}, \mathcal{O}\right)$, where $K^{\prime} \supset \hat{K}$ is a compact set containing a CR submanifold of dimension $\operatorname{dim}_{R} M+e+k-l$. To see this, observe that $\operatorname{dim}_{R} \hat{M}=\operatorname{dim}_{R} M+k-l$, and that the $e$ for $\hat{M}$ is the same as the $e$ for $M$, for Lemma 2.2.1 says that $H(M)$ $=H(\hat{M})$. Next, we prove that the sequence $\left\{g_{j}\right\}$ converges uniformly on $K^{\prime}$. This is due to the fact that the restriction mapping $\Gamma\left(K^{\prime}, \mathcal{O}\right) \rightarrow \Gamma(\hat{K}, \mathcal{O})$ being one-to-one and onto, we must have $\sup _{K^{\prime}}\|f\|=\sup _{\hat{K}}\|f\|$ for every $f \in \Gamma\left(K^{\prime}, \mathcal{O}\right)$. (Otherwise, if $\zeta \in K^{\prime}-\hat{K}$ and $|f(\zeta)|>\sup _{\hat{K}}\|f\|$, the function $(f-f(\zeta))^{-1} \mid \hat{K}$ belongs to $\Gamma(\hat{K}, \mathcal{O})$ 
and is not in the image of $\Gamma\left(K^{\prime}, \mathcal{O}\right)$ by the restriction map.) Hence $\left\{g_{j}\right\}$ is Cauchy on $K^{\prime}$, and it is obvious that its limit $g$ is equal to $\hat{f}$ on $\hat{K}$, and hence to $f$ on $K$. Q.E.D.

Observe that $\mathrm{CR}$ extendibility being a stronger condition than extendibility, we have in particular proved that Theorem 4.2.1 holds without the conditions of genericity and $\operatorname{dim}_{R} M \geqq n$, as long as $M$ satisfies at least one of the conditions (4.1.1). Using the arguments in [5], one can also prove that if $M$ satisfies (I) or (II) of (4.1.1), then it is not locally extendible to a set containing a manifold of real dimension bigger than $\operatorname{dim}_{R} M+e+k-l$.

As an immediate consequence of Theorem 4.2.2, we get the

COROLlaRY 4.2.3. If $M$ satisfies at least one of the conditions of (4.1.1) at $z_{0} \in M$, then there will be a fundamental system of neighborhoods $\{K\}$ of $z_{0}$ in $M$ such that each $K$ is $C R$ extendible to a set containing an open set $U$ in $C^{n}$ ( $U$ depending on $K$ ), if and only if $M$ is generic at $z_{0}$, and $e$ is as big as possible, i.e., $e=l$ $=\operatorname{dim}_{R} M-2 \mathrm{CR} \operatorname{dim} M$.

\section{REFERENCES}

1. A. Andreotti and $\mathrm{H}$. Grauert, Théorèmes de finitude pour la cohomologie des espaces complexes, Bull. Soc. Math. France 90 (1962), 193-259. MR 27 \#343.

2. A. Andreotti and E. Vesentini, Carleman estimates for the Laplace-Beltrami equation on complex manifolds, Inst. Hautes Études Sci. Publ. Math. No. 25 (1965), 81-130. MR 30 \#5333.

3. E. Bishop, Differentiable manifolds in complex Euclidean space, Duke Math. J. 32 (1965), 1-21. MR 34 \#369.

4. S. Greenfield, Cauchy-Riemann equations in several variables, Ann. Scoula Norm. Sup. Pisa (3) 22 (1968), 275-314. MR 38 \#6097.

5. - Upper bounds on the dimension of extendibility of submanifolds in $C^{n}$, Proc. Amer. Math. Soc. 23 (1969), 185-189. MR 41 \#3817.

6. L. Hörmander, $L^{2}$ estimates and existence theorems for the $\bar{\partial}$ operator, Acta Math. 113 (1965), 89-152. MR 31 \#3691.

7. —_, An introduction to complex analysis in several variables, Van Nostrand, Princeton, N. J., 1966. MR 34 \#2933.

8. J. J. Kohn and H. Rossi, On the extension of holomorphic functions from the boundary of a complex manifold, Ann. of Math. (2) 81 (1965), 451-472. MR 31 \#1399.

9. H. Lewy, On the local character of the solutions of an atypical linear differential equation in three variables and a related theorem for regular functions of two complex variables, Ann. of Math. (2) 64 (1956), 514-522. MR 18, 173.

10. — On hulls of holomorphy, Comm. Pure Appl. Math. 13 (1960), 587-591. MR 27 \#340.

11. B. Malgrange, Ideals of differentiable functions, Studies in Math., Tata Institute of Fundamental Research, Bombay.

12. R. Nirenberg, Function algebras on a class of pseudoconvex submanifolds of $\boldsymbol{C}^{n}$, Bull. Un. Mat. Ital. (4) 3 (1970), 628-636. MR 42 \#3306.

13. R. Nirenberg and R. O. Wells, Jr., Approximation theorems on differentiable submanifolds of a complex manifold, Trans. Amer. Math. Soc. 142 (1969), 15-25. MR 39 \#7140.

14. H. Rossi, Holomorphically convex sets in several complex variables, Ann. of Math. (2) 74 (1961), 470-493. MR 24 \#A3310. 
15. H. Rossi, Report in the Proceedings of the International Congress of Mathematicians (Moscow, 1966), “Mir", Moscow, 1968.

16. G. Tomassini, Tracce delle funzioni olomorfe sulle sottovarietà analitiche reali d'una varietà complessa, Ann. Scuola Norm. Sup. Pisa (3) 20 (1966), 31-43. MR 34 \#6808.

17. B. Weinstock, On holomorphic extension from real submanifolds of complex Euclidean space, Thesis, Massachusetts Institute of Technology, Cambridge, Mass., 1966.

18. R. O. Wells, Jr., On the local holomorphic hull of a real submanifold in several complex variables, Comm. Pure Appl. Math. 19 (1966), 145-165. MR 33 \#5948.

19. - Holomorphic hulls and holomorphic convexity, Proc. Conference Complex Analysis (Rice University, Houston, Texas, 1967), Rice Univ. Studies 54 (1968), no. 4, 75-84. MR 39 \#3029.

Department of Mathematics, University of Wisconsin, Madison, Wisconsin 53706

Current address: Department of Mathematics, State University of New York, Albany, New York 12203. 